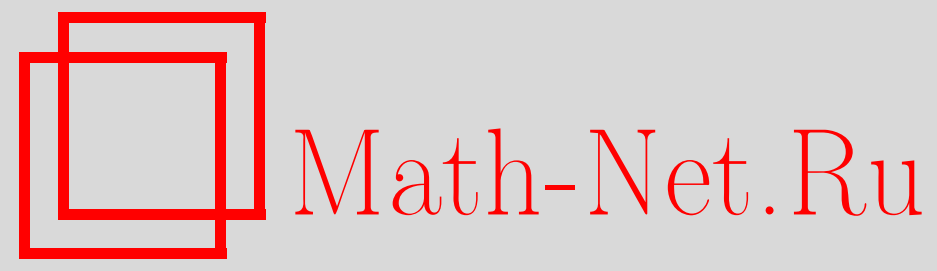

Л. В. Розовский, Одно обобщение теоремы Колмогорова о законе повторного логарифма, Теория вероятн. и ее примен., 1997, том 42, выпуск 1, 134-143

DOI: https://doi.org/10.4213/tvp1716

Использование Общероссийского математического портала Math-Net.Ru подразумевает, что вы прочитали и согласны с пользовательским соглашением http://www.mathnet.ru/rus/agreement

Параметры загрузки:

IP: 107.22 .136 .117

26 апреля 2023 г., 12:37:10

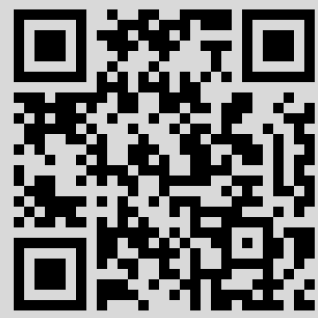


(C) $1997 \mathrm{r}$.

РозовСКИй Л. В.*

\section{ОДНО ОБОБЩЕНИЕ ТЕОРЕМЫ КОЛМОГОРОВА О ЗАКОНЕ ПОВТОРНОГО ЛОГАРИФМА'}

В заметке найдены необходимые и достаточные условия выполнения закона повторного логарифма для суммы определенным образом ограниченных случайных величин в случае произвольной монотонно растушей к бесконечности нормировки. При стандартной нормируюшей последовательности из полученных результатов вытекает известная теорема Колмогорова.

Ключевые слова и фразы: независимые ограниченные случайные величины, закон повторного логарифма, сходимость почти наверное.

1. Введение. Основные результаты. Пусть $\left\{X_{i}\right\}_{i=1}^{\infty}-$ последовательность независимых случайных величин с нулевыми средними и конечными дисперсиями $\sigma_{i}^{2}(i \geqslant 1)$. Положим

$$
S_{n}=X_{1}+\cdots+X_{n}, \quad B_{n}^{2}=\sigma_{1}^{2}+\cdots+\sigma_{n}^{2}, \quad n \geqslant 1 .
$$

Согласно известной теореме А. Н. Колмогорова (см. [1]), если

$$
\text { ess sup }\left|X_{n}\right|=o\left(\frac{b_{n}}{\log \log b_{n}}\right), \quad n \rightarrow \infty,
$$

где $b_{n}=B_{n} \sqrt{2 \log \log B_{n}}$ растет к бесконечности вместе с $n$, то

$$
\limsup _{n \rightarrow \infty} \frac{S_{n}}{b_{n}}=1 \text { п.н. }
$$

Условие (1) является точным: в случае замены «о-малого» в (1) на «O-большое» можно лишь утверждать (см. примеры в [2]-[4]), что

$$
0<\limsup _{n \rightarrow \infty} \frac{S_{n}}{b_{n}}<\infty \quad \text { п.н. }
$$

или, как в [5], что

$$
0<\limsup _{n \rightarrow \infty} \frac{S_{n}}{b_{n}} \leqslant M \quad \text { п.н., }
$$

*Химико-фармацевтическая академия, кафедра математики, ул. проф. Попова, 14, 197376 С.-Петербург, Россия.

1) Работа выполнена при поддержке Российского фонда фундаментальных исследований, грант № 95-01-01260. 
где $M=1+\left(e^{\nu}-1-\nu-\nu^{2} / 2\right) \nu^{-2}$ и

$$
\nu=\limsup _{n \rightarrow \infty} \operatorname{ess} \sup \left|X_{n}\right| \frac{\sqrt{2 \log \log B_{n}}}{B_{n}} \quad(\nu<\infty) .
$$

Основной целью настоящей статьи является распространение фундаментальной теоремы Колмогорова на более общий класс нормирующих постоянных $\left\{b_{n}\right\}$.

Надо сказать, что имеются результаты (см. [6]-[12], например), в том или ином смысле более общие, чем те, что получены в настоящей работе, однако критерии, в них формулирующиеся, либо носят чересчур обший характер, либо слишком сложны.

Введем некоторые обозначения, необходимые для формулировки результатов. Пусть $\left\{b_{n}\right\}$ - положительная растущая к бесконечности последовательность. Так же, как в [6], для любого фиксированного $c>1$ определим $i_{n}$ как наибольшее целое такое, что $b_{i_{n}} \leqslant c^{n}$. Положим

$$
Q_{r}(\alpha)=\sum_{n \geqslant r} \exp \left\{-\frac{\alpha c^{2 n}}{2\left(B_{i_{n}}^{2}-B_{i_{n-r}}^{2}\right)}\right\},
$$

где $\alpha>0, r=1,2, \ldots$

Теорема 1. Пусть с > 1 u $r \geqslant 1-$ челое. Если

$$
\text { ess sup }\left|X_{n}\right|=O\left(\frac{b_{n}}{\log \log b_{n}}\right), \quad n \rightarrow \infty,
$$

mo: a) $\lim \sup _{n \rightarrow \infty}\left(S_{n} / b_{n}\right)<\infty$ n.н. тогда и только тогда, когда

$$
Q_{r}(\alpha)<\infty \quad \text { при некотором } \alpha>0 ;
$$

b) $\lim \sup _{n \rightarrow \infty}\left(S_{n} / b_{n}\right) \leqslant 0$ n.н. тогда и только тогда, когда

$$
Q_{r}(\alpha)<\infty \quad \text { при пюбом } \alpha>0 \text {. }
$$

Таким образом, соотношение (3) справедливо тогда и только тогда, когда удовлетворяются соотношения (6) и

$$
Q_{r}(\beta)=\infty \quad \text { при некотором } \beta>0 .
$$

Благодаря симметрии условия (5) величины $S_{n}$ в теореме 1 можно заменить на $\left|S_{n}\right|$. В частности, имеет место следующий результат.

Следствие 1. Пусть $c>1, r \geqslant 1$ u имеет место условие (5). Tоәда $S_{n} / b_{n} \rightarrow 0$ n.н., если и только если выполняется соотношение (7).

Отметим, что случай $b_{n}=n, c=2, r=1$ был рассмотрен в [13].

Теорема 2. Если выполняется условие (1), то

$$
\limsup _{n \rightarrow \infty} \frac{S_{n}}{b_{n}} \leqslant 1 \quad(<1) \quad \text { n. } .
$$

тогда и только тогда, когда $Q_{r}(\alpha)<\infty$ при любом $\alpha>1$ (при некотором $\alpha<1$ ), пюбом $c>1$ июбом $r \geqslant 1$. 
В частиости, соотношение (2) инеет место тогда и только тогдa, когда $Q(\alpha)<\infty(\forall \alpha>1, \forall c>1 \forall r \geqslant 1) u Q_{r}(\beta)=\infty(\forall \beta<1 \exists c>1$ $\exists r \geqslant 1)$.

Разумеется, сказанное, как это было ранее в теореме 1 , остается справедливым при замене $S_{n}$ на $\left|S_{n}\right|$.

В заключение приведем условия, при которых соотношения типа $Q_{r}(\alpha)<\infty$ сушественно упрошаются.

За м е ч а н и е 1. Предположим, что $b_{b} / B_{n}$ возрастает и либо

$$
\liminf _{n \rightarrow \infty} \frac{B_{n+1}}{B_{n}}=\infty \text {, }
$$

либо

$$
\limsup _{n \rightarrow \infty} \frac{B_{n+1}}{B_{n}}<\infty
$$

и, кроме того,

$$
\frac{B_{n+1}}{B_{n}} \geqslant g\left(\frac{b_{n+1}}{b_{n}}\right) \text { для всех достаточно больших } n,
$$

где $g(x)$ - положительная функция такая, что $g \rightarrow \infty$ и $g(x) g(y) \geqslant g(x y)$ при всех $x, y \geqslant 1$. Тогда $Q_{r}(\alpha)$ в теоремах 1,2 можно заменить на $\sum_{n \geqslant 1} \exp \left\{-\alpha b_{n}^{2} /\left(2 \sigma_{n}^{2}\right)\right\}$ или, соответственно, на

$$
\sum_{n \geqslant 1} \frac{b_{n+1}-b_{n}}{b_{n}} \exp \left\{-\frac{\alpha b_{n}^{2}}{2 B_{n}^{2}}\right\}
$$

Заметим, что условие (12) слабее предположения

$$
\frac{b_{n}}{B_{n}^{\delta}} \quad \text { убывает при некотором } \delta>1
$$

(и совнадает с ним при $g(x)=x^{\delta}$ ).

Очевидно также, что ряд в (13) сходится при $\alpha>1$ и расходится при $\alpha<1$, если $b_{n}=B_{n} \sqrt{2 \log \log B_{n}}$. Следовательно, критерии для (2) и (3) в этом случае вытекают из теорем 1 и 2.

\section{2. Вспомогательные результаты и доказательства.}

Лемма 1. Пусть $Y_{1}, \ldots, Y_{n}(n \geqslant 1)$ - независимые случайные величияы такие, ито $\left|Y_{i}\right| \leqslant C$ п.н., $\mathbf{E} Y_{i}^{2}<\infty, \mathbf{E} Y_{i}=0$, где $1 \leqslant i \leqslant n, a$ $C$ - некоторая положительная постоянная. Пусть, кроме того,

$$
\begin{aligned}
Z_{n} & =Y_{1}+\cdots+Y_{n}, \quad D_{n}^{2}=\mathrm{E} Z_{n}^{2}, \\
\omega & =\omega(\beta)=\left(2 \beta^{-2}\left(e^{\beta}-1-\beta\right)\right)^{-1 / 2}, \quad \beta>0 .
\end{aligned}
$$

Tozda:

$$
\begin{array}{lr}
\mathbf{P}\left\{Z_{n} \geqslant x\right\} \leqslant \exp \left\{-\frac{x^{2}}{2 D_{n}^{2} \omega^{2}}\right\}, & 0 \leqslant x \leqslant \beta \omega^{2} \frac{D_{n}^{2}}{C} \\
\mathbf{P}\left\{Z_{n} \geqslant x\right\} \leqslant \exp \left\{-\frac{\beta x}{2 C}\right\}, & x \geqslant \beta \omega^{2} \frac{D_{n}^{2}}{C}
\end{array}
$$


еслu $\beta \in\left(0,10^{-2}\right) u 0<C \leqslant \beta^{2} D_{n}$, mo

$$
\mathbf{P}\left\{Z_{n} \geqslant x\right\} \geqslant \exp \left\{-\frac{(1+50 \beta) x^{2}}{2 D_{n}^{2}}\right\}, \quad \beta^{-1} D_{n} \leqslant x \leqslant \frac{\beta D_{n}^{2}}{C}
$$

кроме того, при всех $x$

$$
\left|\mathbf{P}\left\{Z_{n}<x\right\}-\Phi\left(\frac{x}{D_{n}}\right)\right| \leqslant \frac{K_{0} C}{|x|},
$$

әде $\Phi(\cdot)$ - стандартная нормальная функция распределения, $K_{0}-a 6-$ солютная постоянная.

Заметим, что неравенства $(14),(15)$ уточняют соответствующие неравенства Колмогорова (см. [1], а также [14, гл. 10]). Отметим также, что оценки (14) близки к известному неравенству Беннета-Хёфдинга [18], [19], а аналог неравенства (15) можно извлечь из результатов работы [20].

Д о к а з а т е л ь с т в о. Проверим сначала соотношение (16). Используя неравномерный вариант теоремы Эссеена [15], найдем, что при некоторой абсолютной постоянной $K_{1}$

$$
\begin{aligned}
\left|\mathbf{P}\left\{Z_{n}<x D_{n}\right\}-\Phi(x)\right| & \leqslant K_{1}(1+|x|)^{-3} D_{n}^{-3} \sum_{j=1}^{n} \mathbf{E}\left|Y_{j}\right|^{3} \\
& \leqslant K_{1} C(1+|x|)^{-3} D_{n}^{-1}=K_{1} C|x|^{-1}\left(\tau+\tau^{-2}\right)^{-1}
\end{aligned}
$$

где $\tau=D_{n} /|x|$. Принимая во внимание то, что $\max _{\tau>0}\left(\tau+\tau^{-2}\right)^{-1}=$ $2^{2 / 3} / 3$, получим соотношение $(16)$.

Для доказательства (14) используем следующие аргументы. ПолоЖИМ

$$
L_{k}(h)=\mathbf{E} \exp \left\{h Y_{k}\right\}, \quad h \geqslant 0, \quad(1 \leqslant k \leqslant n) .
$$

Применяя неравенство Чебышева, легко получим оценку

$$
\mathbf{P}\left\{Z_{n} \geqslant x\right\} \leqslant \exp \left\{-h x+\sum_{k=1}^{n}\left(L_{k}(h)-1\right)\right\} \text {. }
$$

Пусть $h=\min \left(\beta / C, x / D_{n}^{2} \omega^{2}\right)$. Функция $\omega(\beta)$ монотонно возрастает, и, следовательно,

$$
\begin{aligned}
\sum_{k=1}^{n}\left(L_{k}(h)-1\right) & =\sum_{k=1}^{n} \mathbf{E}\left(\exp \left\{h Y_{k}\right\}-1-h Y_{k}\right)=\frac{1}{2} h^{2} \sum_{k=1}^{n} \mathbf{E} \omega^{2}\left(h Y_{k}\right) Y_{k}^{2} \\
& \leqslant \frac{1}{2} h^{2} \omega^{2}(h C) D_{n}^{2} \leqslant \frac{1}{2} h^{2} \omega^{2} D_{n}^{2}
\end{aligned}
$$

Отсюда и из (18) вытекают соотношения (14).

Проверка оценки (15) несколько сложнее. Положим $\eta=10 \beta, \bar{\gamma}=$ $\beta \sqrt{2}, \delta=\beta \sqrt{1.5}, \gamma=3 \beta^{2}\left(0<\beta<10^{-2}\right)$ и выберем $h=(1+\eta) x / D_{n}^{2}$, где $\beta^{-1} D_{n} \leqslant x \leqslant \beta D_{n}^{2} / C$. 
Имеем (см. (17), [14, гл. 8]):

$$
\mathbf{P}\left\{Z_{n} \geqslant x\right\}=\left[\prod_{k=1}^{n} L_{k}(h)\right] \int_{x}^{\infty} e^{-h y} d \mathbf{P}\left\{\bar{S}_{n}<y\right\},
$$

где $\bar{S}_{n}=\sum_{k=1}^{n} \bar{Y}_{k}$ и $\left\{\bar{Y}_{k}\right\}$ - независимые случайные величины с функциями распределения $\left(1 / L_{k}(h)\right) \mathbf{E} \exp \left\{h Y_{k}\right\} \mathbf{I}\left\{Y_{k}<y\right\}$.

Вычисления показывают, что

$$
\begin{aligned}
& L_{k}(h)=1+\frac{1}{2} h^{2}\left(1+\frac{1}{6} \theta_{1}\right) \mathbf{E} Y_{k}^{2}, \\
& L_{k}^{\prime}(h)=h\left(1+\frac{1}{2} \theta_{2}\right) \mathbf{E} Y_{k}^{2}, \quad L_{k}^{\prime \prime}(h)=\left(1+\theta_{3}\right) \mathbf{E} Y_{k}^{2},
\end{aligned}
$$

где $\left|\theta_{i}\right| \leqslant \delta e^{\delta}, i=1,2,3 ;$ кроме того,

$$
\left|L_{k}(h)-1\right| \leqslant \frac{1}{2} h^{2} e^{h C} \mathbf{E} Y_{k}^{2} \leqslant \frac{1}{2} \delta^{2} e^{\delta}
$$

(напоминаем, что $h C<\delta$ при $x \leqslant \beta D_{n}^{2} / C$ ).

Обозначим множитель в правой части (19) через $A_{1}$, а интеграл через $A_{2}$. Таким образом,

$$
\mathbf{P}\left\{Z_{n} \geqslant x\right\}=A_{1} A_{2} .
$$

Оценим $A_{1}$ снизу. В соответствии с (21), (20)

$$
\begin{aligned}
\log L_{k}(h) & \geqslant L_{k}(h)-1-\frac{\gamma}{2(1-\gamma)^{2}}\left|L_{k}(h)-1\right| \\
& \geqslant \frac{1}{2} h^{2} D_{n}^{2}\left(1-\frac{1}{4} \beta\right)-\frac{\gamma}{4(1-\gamma)^{2}} h^{2} e^{\delta} D_{n}^{2} \geqslant \frac{1}{2} h^{2} D_{n}^{2}(1-0.4 \beta) .
\end{aligned}
$$

Следовательно,

$$
A_{1} \geqslant \exp \left\{\frac{1}{2}(1-0.4 \beta) h^{2} D_{n}^{2}\right\}
$$

Далее,

$$
A_{2}=e^{-h x} \int_{0}^{\infty} e^{-h t} d \mathbf{P}\left\{\bar{S}_{n}<t+x\right\} \geqslant e^{-h(1+2 \eta) x}\left(1-P_{2}\right),
$$

где $P_{2}=\mathbf{P}\left\{\bar{S}_{n}>(1+2 \eta) x \cup \bar{S}_{n}<x\right\}$.

Имеем

$$
\mathbf{E} \bar{S}_{n}=\sum_{k=1}^{n}\left(L_{k}^{\prime}(h)+L_{k}^{\prime}(h) \frac{1-L_{k}(h)}{L_{k}(h)}\right)=J_{1}+J_{2},
$$

причем в согласии с (20), (21)

$$
J_{1}=\left(1+\theta_{4}\right) h D_{n}^{2}, \quad\left|\theta_{4}\right| \leqslant \frac{1}{2} \delta e^{\delta}<0.7 \beta ; \quad J_{2} \leqslant 0.04 \beta .
$$


Из (25), (26) следует, что $9 \beta \leqslant\left(\mathbf{E} \bar{S}_{n}-x\right) / x \leqslant 11 \beta$, и, значит, (см. также (20)) при $\beta^{-1} D_{n} \leqslant x$

$$
P_{2} \leqslant(9 \beta x)^{-2} \operatorname{Var} \bar{S}_{n} \leqslant 2\left(1+\delta e^{\delta}\right) \beta^{-2} \frac{D_{n}^{2}}{81 x^{2}}<1-e^{-1} \leqslant 1-e^{-\beta^{2} x^{2} / D_{n}^{2}} .
$$

Отсюда и из (22)-(24) (напоминаем, что $\beta \in\left(0,10^{-2}\right)$ ) легко получим оценку (15).

Лемма 1 полностью доказана.

Лемма 2. Пусть $\left\{U_{n}, n \geqslant 1\right\}$ - последовательность случайных величин, $\left\{a_{n}, n \geqslant 1\right\}$ - положительная иисловая последовательность. Положим $U_{0}=0 u a_{0}=0$. Тогда

$$
\mathbf{P}\left\{\sup _{k \geqslant 1} \frac{U_{k}}{\boldsymbol{a}_{k}} \geqslant 1\right\} \leqslant \sum_{k \geqslant 0} \mathbf{P}\left\{U_{k+1}-U_{k} \geqslant a_{k+1}-a_{k}\right\} .
$$
Имеем:

Д ок а з а те льст во лем мы 2 проводится аналогично [16].

$$
\left\{\sup _{k \geqslant 1} \frac{U_{k}}{a_{k}} \geqslant 1\right\}=\bigcup_{k=0}^{\infty} B_{k}
$$

где

$$
B_{0}=\left\{U_{1} \geqslant a_{1}\right\}, \quad B_{k}=\left\{\sup _{1 \leqslant m \leqslant k} \frac{U_{m}}{a_{m}}<1, \frac{U_{k+1}}{a_{k+1}} \geqslant 1\right\}, \quad k \geqslant 1 .
$$

При этом $B_{m} \cap B_{k}=\varnothing$, если $m \# k$. Остается заметить, что при $k \geqslant 1$

$$
\begin{aligned}
B_{k} & \subset\left\{\frac{U_{k}}{a_{k}}<1, \frac{U_{k+1}-U_{k}}{a_{k+1}}+\frac{a_{k}}{a_{k+1}} \frac{U_{k}}{a_{k}} \geqslant 1\right\} \\
& \subset\left\{\frac{U_{k+1}-U_{k}}{a_{k+1}}+\frac{a_{k}}{a_{k+1}} \geqslant 1\right\} .
\end{aligned}
$$

Лемма 3. Пусть при всех достаточно больиих $n$

$$
\left|X_{n}\right| \leqslant \frac{\gamma b_{n}}{\log \log b_{n}} \quad \text { n.н., }
$$

где $\gamma$ - некоторая положительная постоянная; $c>1, r \geqslant 1-$ челое, $\beta \in\left(0,10^{-2}\right), 0<M<\infty-$ некоторые числа. Тогда если

$$
\limsup _{n \rightarrow \infty} \frac{S_{n}}{b_{n}} \leqslant M \quad n . \mu .
$$

mо $Q_{r}(\alpha)<\infty$ при любом $\alpha>\max \left(2 \gamma M \beta^{-1}, M^{2}(1+50 \beta)\right)$.

Д о к а з а те ль с т в о. Положим

$$
A_{m}=\left\{S_{i_{\bar{m}}}-S_{i_{\bar{m}-r}}\right\}>\bar{M} c^{\bar{m}}, \quad B_{m}=\left\{S_{i_{\bar{m}-r}}>-\varepsilon c^{\bar{m}}\right\},
$$

где $\bar{m}=m r+j(0 \leqslant j<r), \bar{M}=M+2 \varepsilon, \varepsilon>0$. 
Из (28) следует, что $0=\mathbf{P}\left\{S_{n}>\bar{M} b_{n}\right.$ б.ч. $\} \geqslant \mathbf{P}\left\{A_{m} B_{m}\right.$ б.ч. $\}$. Далее, при помоши леммы 1 (оценка (16)) и соотношения (27) получим

$$
\mathbf{P}\left\{B_{m}\right\} \geqslant \frac{1}{2}-K_{0}\left(\gamma c^{\bar{m}} / \log \log c^{\bar{m}}\right) / \varepsilon c^{\bar{m}}>\frac{1}{3},
$$

если $\boldsymbol{m}$ достаточно велико.

Из сказанного следует (см. [17]), что ряд $\sum_{m} \mathbf{P}\left\{A_{m}\right\}$ сходится. Покажем, что при всех достаточно больших $\boldsymbol{m}$

$$
\left(\frac{1}{m}\right)^{1+\varepsilon}+\mathbf{P}\left\{A_{m}\right\} \geqslant \exp \left\{-\frac{\tau c^{2 \bar{m}}}{2 \Delta_{m}^{2}}\right\}
$$

где $\Delta_{m}^{2}=\mathbf{E}\left(S_{i_{\bar{m}}}-S_{i_{\bar{m}-r}}\right)^{2}, \tau=\max \left\{(1+50 \beta) \bar{M}^{2},(2+2 \varepsilon) \bar{\gamma} \bar{M} \beta^{-1}\right\}$ и $\bar{\gamma}=(1+\varepsilon) \gamma$.

Если показатель экспоненты в $(29)$ больше, чем $(1+\varepsilon) \log \bar{m}$, соотношение (29) выполняется. Поэтому далее будем предполагать, что

$$
\frac{c^{\bar{m}}}{\sqrt{\log \bar{m}}} \leqslant \Delta_{m} \sqrt{\frac{2+2 \varepsilon}{\tau}}
$$

С помощью опенки (15) леммы 1 , сделав подходящие переобозначения, и имея в виду, что $|\log \log c / \log \bar{m}|<\varepsilon /(1+\varepsilon)$, если $\bar{m}$ достаточно велико, заклточаем:

$$
\mathbf{P}\left\{A_{m}\right\} \geqslant \exp \left\{-\frac{\tau c^{2 \bar{m}}}{2 \Delta_{m}^{2}}\right\}
$$

если

$$
\bar{\gamma} \frac{c^{\bar{m}}}{\log \bar{m}}<\beta^{2} \Delta_{m}, \quad c^{\bar{m}} \geqslant(\beta \bar{M})^{-1} \Delta_{m}, \quad \frac{c^{\bar{m}}}{\sqrt{\log \bar{m}}} \leqslant \Delta_{m} \sqrt{\frac{\beta}{\bar{\gamma} \bar{M}}}
$$

(напоминаем, что $\tau \geqslant(1+50 \beta) \bar{M}^{2}$ ).

Из оценки (16) леммы 1 вытекает неравенство $1-\Phi\left(\bar{M} c^{\bar{m}} / \Delta_{m}\right) \leqslant$ $\mathbf{P}\left\{A_{m}\right\}+k_{0} \bar{\gamma} / \log \bar{m}$, из которого следует, что $c^{\bar{m}} / \Delta_{m} \rightarrow \infty$ при $m \rightarrow \infty$. Справедливость второй оценки в (32), таким образом, установлена.

Первая и третья оценки в (32) следуют из (30), поскольку $\beta /(\bar{\gamma} \bar{M}) \geqslant$ $(2+2 \varepsilon) \tau^{-1}$ по выбору $\tau$. Итак, оценка (29) доказана.

Из (29) при $j=0, \ldots, r-1$ следует, что $Q_{t}(\tau)<\infty$. В свою очередь отсюда в силу произвольности $\varepsilon>0$ вытекает утверждение леммы 3 .

Лемма 4. Пусть выполняется условие (27); $>>1, r>1$ - челое, $\beta>0, \alpha>0$ - некоторые числа. Положим $M_{0}=\max \left(\alpha \omega, 2 \gamma \beta^{-1}\right) c /(1-$ $\left.c^{-r}\right)$, где $\omega=\omega(\beta)$ определено в лемме 1 . Тогда если $Q_{r}\left(\alpha^{2}\right)<\infty$, то

$$
\limsup _{n \rightarrow \infty} \frac{S_{n}}{b_{n}} \leqslant M_{0} \quad \text { n.н. }
$$


Д о к а з а т е л ь с т в о. Пусть (см. обозначения перед теоремой 1) $\bar{b}_{j}=c^{n}, i_{n-1}<j \leqslant i_{n}, n=1,2, \ldots ; \bar{M}=M_{0}(1+2 \varepsilon), \varepsilon>0$. Имеем

$$
\begin{aligned}
P_{m}(\bar{M}) & =\mathbf{P}\left\{\sup _{l \geqslant i_{m}} \frac{S_{l}}{b_{l}} \geqslant \bar{M}\right\} \leqslant \mathbf{P}\left\{\sup _{l \geqslant i_{m}} \frac{S_{l}}{\bar{b}_{l}} \geqslant \bar{M} c^{-1}\right\} \\
& \leqslant \sum_{j=0}^{r-1} P_{m, j}(\widetilde{M}),
\end{aligned}
$$

где $\widetilde{M}=\bar{M} c^{-1}, P_{m, j}(\widetilde{M})=\mathbf{P}\left\{\sup _{k \geqslant 0} T_{k, j} / c^{k r+m+j} \geqslant \widetilde{M}\right\}$ и

$$
T_{k, j}=\max _{i_{k r+j-1+m}<l \leqslant i_{k r+j+m}} S_{n} \text { для } k \geqslant 1, \quad T_{0, j}=S_{i_{m}+j} .
$$

Оценим $P_{m, j}(\widetilde{M}), 0 \leqslant j<r$. Пусть для определенности $j=0$. Учитывая, что $T_{k, 0} \geqslant S_{i_{k r+m}}$ п.н., из леммы 2 получим

$$
\begin{gathered}
P_{m, 0}(\widetilde{M}) \leqslant \mathbf{P}\left\{S_{i_{m}} \geqslant \widetilde{M} c^{m}\right\}+\sum_{k \geqslant 1} \mathbf{P}\left\{\max _{i_{k r-r+m}<l \leqslant i_{k r+m}}\left(S_{l}-S_{i_{k r-r+m}}\right)\right. \\
\left.\geqslant \widetilde{M} c^{k r+m}\left(1-c^{-r}\right)\right\} .
\end{gathered}
$$

Принимая во внимание условие $Q_{r}\left(\alpha^{2}\right)<\infty$, из которого следует, что $\mathbf{E}\left(S_{i_{k r+m}}-S_{i_{k r-r+m}}\right)^{2}=o\left(c^{k r+m}\right), k r+m \rightarrow \infty$, и неравенство Колмогорова (см. $[14$, гл. 3$])$, из (35) при всех достаточно больших $m$ получим оценку

$$
P_{m, 0}(\widetilde{M}) \leqslant \mathbf{P}\left\{S_{i_{m}} \geqslant \widetilde{M} c^{m}\right\}+2 \sum_{k \geqslant 1} \mathbf{P}\left\{S_{i_{k r+m}}-S_{i_{k r+r+m}} \geqslant \widetilde{M} c^{k r+m}\right\},
$$

где $\widehat{M}=\left(1-c^{-r}\right) c^{-1} M_{0}(1+\varepsilon)$.

Обозначим $k$-ю вероятность из суммы в $(36)$ через $\bar{P}_{k}(\widehat{M})$ и положим $D_{k}^{2}=B_{i_{k r+m}}^{2}-B_{i_{k r-r+m}}^{2}$. В соответствии с леммой 1 (оценки (14)), условием (27) и определением $M_{0}$,

$$
\begin{aligned}
\bar{P}_{k}(\widehat{M}) & \leqslant \exp \left\{-\frac{\widehat{M}^{2} c^{2(k r+m)}}{2 \omega^{2} D_{k}^{2}}\right\}+\exp \left\{-\frac{\beta \widehat{M}}{2 \gamma} \log \log c^{2(k r+m)}\right\} \\
& \leqslant \exp \left\{-\frac{(1+\varepsilon)^{2} \alpha^{2} c^{2(k r+m)}}{2 D_{k}^{2}}\right\}+((k r+m) \log c)^{-(1+\varepsilon)}
\end{aligned}
$$

Отсюда, из (36), (34) в силу произвольности $\varepsilon$ следует утверждение леммы 4.

Теоремы 1 и 2 без каких-либо затруднений можно доказать с помощью лемм 3 и 4.

Установим справедливость замечания 1. Положим

$$
T(\alpha)=\sum_{n \geqslant 1} \frac{b_{n+1}-b_{n}}{b_{n}} \exp \left\{-\frac{\alpha b_{n}^{2}}{2 B_{n}^{2}}\right\}
$$


Очевидно, в силу определения $\left\{i_{n}\right\}$ при любых $c>1$ и $r \geqslant 1$

$$
\begin{aligned}
2 T(\alpha) & \geqslant \sum_{n \geqslant 1} \sum_{j=i_{n-1}}^{i_{n}} \frac{b_{j+1}-b_{j}}{b_{j}} \exp \left\{-\frac{\alpha b_{j}^{2}}{2 B_{j}^{2}}\right\} \\
& \geqslant \sum_{n \geqslant 1} \frac{c^{n}-c^{n-1}}{c^{n}} \exp \left\{-\frac{\alpha b_{i_{n}}^{2}}{2 B_{i_{n}}^{2}}\right\} \geqslant\left(1-c^{-1}\right) Q_{r}(\alpha) .
\end{aligned}
$$

Предположим теперь, что выполняются условия (11), (12). Тогда (напоминаем, что $\left.\lim _{y \rightarrow \infty} g(y)=\infty\right) b_{n+1} / b_{n} \leqslant K$ при некотором $K>1$ и всех достаточно больших $n$. Далее, по (12)

$$
\frac{B_{i_{n}}}{B_{i_{n-r}}} \geqslant g\left(\frac{b_{i_{n}}}{b_{i_{n-r}}}\right)=g\left(\frac{b_{i_{n}+1}}{b_{i_{n-r}}} \frac{b_{i_{n}}}{b_{i_{n}+1}}\right) \geqslant g\left(\frac{c^{n}}{c^{n-r}} \frac{1}{K}\right)=g\left(\frac{c^{r}}{K}\right),
$$

и, следовательно, при любом $\varepsilon \in(0,1)$ можно добиться справедливости неравенства $B_{i_{n-r}}^{2} \leqslant \varepsilon B_{i_{n}}^{2}$, выбирая, скажем, $c^{r} \geqslant M_{\varepsilon}$. В этом случае

$$
\begin{aligned}
T\left(\frac{\alpha c^{2}}{1-\varepsilon}\right) & \leqslant \sum_{n \geqslant 1} \sum_{j>i_{n-1}}^{i_{n}} \frac{b_{j+1}-b_{j}}{b_{j}} \exp \left\{-\frac{\alpha c^{2}}{2(1-\varepsilon)} \frac{c^{2(n-1)}}{B_{i_{n}}^{2}}\right\} \\
& \leqslant K c Q_{r}(\alpha) .
\end{aligned}
$$

Действительно, если $i_{n-1}<l \leqslant i_{n}$, то $c^{n-1}<b_{l} \leqslant c^{n}$ и $B_{l} \leqslant B_{i_{n}}$; кроме того,

$$
\sum_{l>i_{n-1}}^{i_{n}} \frac{b_{l+1}-b_{l}}{b_{l}} \leqslant \frac{b_{i_{n}+1}}{b_{i_{n-1}+1}} \leqslant \frac{K c^{n}}{c^{n-1}}=K c .
$$

Из (37), (38) следует одно из утверждений замечания (при доказательстве используется то, что выбор $c$ и $r$ в наших руках). Другое утверждение замечания доказывается проше - достаточно заметить, что при условии (10) $B_{n}^{2} \sim \sigma_{n}^{2}$ при $n \rightarrow \infty$.

3. Дополнение. Приведенные выше леммы 3 и 4 позволяют связать величину $\lim \sup _{n \rightarrow \infty}\left(S_{n} / b_{n}\right)$ с параметром $\alpha$ в условиях типа (7), (8). В качестве примера приведем следуюший результат, уточняющий как теорему Колмогорова, так и соответствующее утверждение из [5] (см. (4)).

Теорема 3. ПГусть $B_{n} \rightarrow \infty$. Ecлu

$$
\left|X_{n}\right| \leqslant \frac{\nu B_{n}}{\sqrt{2 \log \log B_{n}}} \quad \text { n.н., }
$$

mo

$$
M_{l} \leqslant \limsup _{n \rightarrow \infty} \frac{S_{n}}{B_{n} \sqrt{2 \log \log B_{n}}} \leqslant M_{u} \quad \text { n.r. }
$$

Здесь $M_{l}=(1+100 \nu)^{-1}, M_{u}=\left(2 \nu^{-2}\left(e^{\nu}-1-\nu\right)\right)^{1 / 2}$.

Отметим, что $M_{l}<l<M_{u}$ и как $M_{l}$, так и $M_{u}$ стремятся к 1 при $\nu \rightarrow 0$. 


\section{СПИСОК ЛИТЕРАТУРЫ}

1. Kolmogoroff $A$. $N$. Über das Gesets des iterierten Logarithmus. - Math. Ann., 1929, v. 101, p. 126-135.

2. Marcienkiewicz $J$., Zygmund A. Remarque sur la loi du logarithme itéré. - Fund. Math., 1937, v. 29, p. 215-222.

3. Weiss $M$. On the law of the iterated logarithm. - J. Math. Mech., 1959, v. 8 (1), p. 121-132.

4. Егоров В. А. К теореме Колмогорова о законе повторного логарифма. - Вестник ЛГУ, 1972, в. 13, с. 140-142.

5. Tomkins R. J. On the law of the iterated logarithm. - Ann. Probab., 1978, v. 6, № 1, p. 162-168.

6. Мартикайнея $А$. И., Петров В. В. О необходимых и достаточных условиях для закона повторного логарифма. - Теория вероятн. и ее примен., 1977, т. XXII, B. 1 , c. $18-26$; в. 2 , c. 442 .

7. Tomkins $R$. J. Limit theorems without moment hypotheses for sums of independent random variables. - Ann. Probab., 1980, v. 8, № 2, p. 314-324.

8. Мартикайнея $А$. И. Три теоремы о верхнем пределе роста сумм незаяисимых случайных величин. - Вестник ЛГУ, 1979, в. 1, с. 45-51.

9. Мартикаймея $A$. И. Экспоненциальный критерий для захона повторного логарифма. - Записки научн. семинаров ЛОМИ, 1979, т. 85, с. 158-168.

10. Нагаев C. B. О необходимых и достаточных условиях для усиленного закона больших чисел. - Теория вероятн. и ее примен., 1972, т. XVII, в. 4, с. 609-618.

11. Мартикайнея А. И. О необходимых и достаточных условиях для усиленного закона больших чисел. - Теория вероятн. и ее примен., 1979, т. XXIV, в. 4, c. 814-821.

12. Володия $H$. А., Нагаев $C$. В. Замечание х усиленному закону больших чисел. Теория вероятн. и ее примен., 1977, т. XXII, в. 4, с. 810-813.

13. Прохоров Ю. В. Несколько замечаний к усиленному закону больших чисел. Теория вероятн. и ее примен., 1959, т. IV, в. 2, с. 204-208.

14. Петров В. В. Суммы независимых случайных величин. М.: Наука, 1972, 416 с.

15. Бикялис $A$. Оценки остаточного члена в центральной предельной теореме. Литов. матем. с6., 1966, т. 6, в. 3, с. 323-346.

16. Розовский $Л$. В. О схорости сходимости в усиленном законе больших чисел. Теория вероятн. и ее примен., 1981, т. XXVI, в. 1, с. 138-142.

17. Baum L. E., Katz M., Stratton H. H. Strong laws for ruled sums. - Ann. Math. Statist., 1971, v. 42, № 2, p. 625-629.

18. Bennet $G$. Probability inequalities for sums of random variables. - J. Amer. Statist. Assoc., 1962, v. 57, № 297, p. 33-45.

19. Hoeffding $W$. Probability inequalities for sums of bounded random variables. J. Amer. Statist. Assoc., 1963, v. 58, № 301, p. 33-45.

20. Архангельский $A$. $H$. Нижние оценки для вероятностей больших уклонений сумм независимых случайных величин. - Теория вероятн. и ее примен., 1989, т. 34, B. 4 , c. $625-635$.

Поступила в редакцию

28.IX.1993

Исправленный вариант

21.VI.1996 